\title{
Doxorubicin hinders DNA condensation promoted by the protein bovine serum albumin (BSA)
}

\author{
C. H. M. Lima ${ }^{1}$ | H. M. C. de Paula ${ }^{2}$ | L. H. M. da Silva ${ }^{2}$ | M. S. Rocha ${ }^{1}$ (10)
}

${ }^{1}$ Departamento de Física, Universidade Federal de Viçosa, Viçosa, Minas Gerais, Brazil

${ }^{2}$ Departamento de Química, Universidade Federal de Viçosa, Viçosa, Minas Gerais, Brazil

\section{Correspondence}

M. S. Rocha, Departamento de Física, Universidade Federal de Viçosa, Viçosa, Minas Gerais, Brazil.

Email: marcios.rocha@ufv.br

\begin{abstract}
In this work, we have studied the interaction between the anticancer drug doxorubicin (doxo) and condensed DNA, using optical tweezers. To perform this task, we use the protein bovine serum albumin (BSA) in the working buffer to mimic two key conditions present in the real intracellular environment: the condensed state of the DNA and the abundant presence of charged macromolecules in the surrounding medium. In particular, we have found that, when doxo is previously intercalated in disperse DNA, the drug hinders the DNA condensation process upon the addition of BSA in the buffer. On the other hand, when bare DNA is firstly condensed by BSA, doxo is capable to intercalate and to unfold the DNA condensates at relatively high concentrations. In addition, a specific interaction between BSA and doxo was verified, which significantly changes the chemical equilibrium of the DNA-doxo interaction. Finally, the presence of BSA in the buffer stabilizes the double-helix structure of the DNA-doxo complexes, preventing partial DNA denaturation induced by the stretching forces.
\end{abstract}

\section{KEYWORDS}

bovine serum albumin (BSA), DNA condensation, doxorubicin, intercalation

\section{1 | INTRODUCTION}

The investigation of DNA interactions with binding drugs is important to understand the physicochemical details of the mechanism(s) of action of such drugs when used in chemotherapies, gene therapies, and other applications. In fact, a number of researchers have paid attention to this topic in the past years, characterizing many types of interactions that can exist between DNA and small drug molecules. For recent reviews on the subject, see Refs. 1,2.

Despite the large number of studies in the field, almost the totality of these works were performed using disperse DNA molecules in ordinary buffer solutions, which contain basically only counter- and co-ions $\left(\mathrm{Na}^{+}, \mathrm{Cl}^{-}\right.$, etc). Although the interactions between DNA and drugs can be characterized in many aspects under such conditions, there is no guarantee that such interactions will occur in the same way inside living cells. On the contrary, they should be distinct in many aspects, since in the intracellular environment the DNA molecules are usually subjected to very different conditions: they are condensed during most time and, in addition, the surrounding medium contains a lot of other macromolecules (proteins, sugars, and others) that contribute to regulate cell volume and fluid balance. Such macromolecules can directly or indirectly interfere in the natural intracellular DNA-related processes and in the DNA-drug interactions, by changing water activity on the double-helix, or even by directly interacting with the biopolymer and/or with the drug used. ${ }^{[3-9]}$ Despite the relevance of such topic, few works have used force spectroscopy to investigate the DNA condensation process at a single molecule level ${ }^{[10-15]}$ and the interactions of condensed DNA with drugs. ${ }^{[14,16-18]}$

In this work, we have studied the interaction between condensed DNA and the drug doxorubicin (doxo) by using the protein bovine serum albumin (BSA) to mimic two key intracellular conditions: DNA condensation and the presence of charged macromolecules in the surrounding medium. Although histones are the real proteins that condense the DNA molecule inside most cells, the use of BSA here has some advantages for letting one to get first insights on the general behavior of the drug inside cells, because the two intracellular conditions above mentioned can be achieved simultaneously by using a single protein in the buffer. Thus, in the present work we created a simplified in vitro environment that somewhat mimics some important intracellular conditions. This environment is closer to what occurs inside prokaryote cells, in which the DNA condensation also depends on the depletants present in the surrounding medium. ${ }^{[19]}$ 
It was recently shown that the BSA protein induces DNA condensation when added at a concentration of $20 \%$ in mass (wt/wt) in a buffer solution with $[\mathrm{NaCl}]=125 \mathrm{mM}^{[15,20]}$ which is the condition chosen the perform the experiments here. Doxo is an anthracycline antibiotic much used in cancer chemotherapies, especially to treat some types of leukemias, sarcomas, lymphomas, myelomas, neuroblastomas, and so forth. The interaction between doxo and disperse DNA has already been characterized in many aspects by using very different techniques such as optical tweezers, ${ }^{[21]}$ magnetic tweezers, ${ }^{[22]}$ microcalorimetry, ${ }^{[23]}$ viscosimetry, ${ }^{[23,24]}$ molecular modeling, ${ }^{[25,26]}$ atomic force microscopy (AFM), ${ }^{[21]}$ dynamic light scattering, ${ }^{[21]}$ crystallography, ${ }^{[2]}$ and various types of spectroscopies. ${ }^{[23-26]}$ Therefore, much information is known about the interaction when using disperse DNA molecules. In the present work, on the other hand, we attempt to investigate the DNA-doxo interaction under experimental conditions closer to the in vivo environment, using the BSA protein to mimic important intracellular conditions. To perform such task, we have used optical tweezers in the low-force entropic regime $(F<3 \mathrm{pN})$ to stretch the DNA-doxo complexes under the desired experimental conditions (phosphate buffered saline (PBS) solution with $[\mathrm{NaCl}]=125 \mathrm{mM}$, $\mathrm{pH}=7.4$, and $\mathrm{BSA}$ at $20 \% \mathrm{wt} / \mathrm{wt})$. The results obtained can be directly compared to the similar ones obtained in Ref. 21 for disperse DNA molecules, thus giving insights on the effects of globular proteins present in the intracellular environment on the DNA interactions with intercalators.

\section{2 | MATERIALS AND METHODS}

The optical tweezers setup and the experimental procedure used here were described in detail elsewhere. ${ }^{[21]}$ Briefly, our optical tweezers consist of a $1064 \mathrm{~nm}$ laser (IPG Photonics) operating in the TEM 00 mode. The laser is mounted on a Nikon Ti-S inverted microscope with a $\times 100$ NA 1.4 objective to form the optical tweezers.

Our samples consist of $\lambda$-DNA molecules (New England Biolabs) in a PBS buffer (4.375 mM Na $2 \mathrm{HPO}_{4}, 1.25 \mathrm{mM} \mathrm{NaH} \mathrm{PO}_{4}, 125 \mathrm{mM}$ $\mathrm{NaCl})$. One end of the DNA molecules is attached to polystyrene beads (Bangs Labs) and the other end to a streptavidin-coated coverslip, using biotin-streptavidin binding in both cases.

A piezoelectric actuator (Physik Instrumente) is used to move the microscope stage and to stretch the DNA molecules. The force-extension curves obtained were fitted to the Marko-Siggia Worm-Like Chain (WLC) model ${ }^{[27]}$ in order to obtain the persistence and contour lengths of the DNA-doxo complexes. In particular, when the DNA molecules are condensed one cannot determine the real contour length from the stretchings performed in the entropic regime, but instead the apparent contour length, since the small forces applied in this regime $(F<3 p N)$ usually are not sufficient to fully stretch the condensed molecule, and therefore the measured (apparent) contour length will be smaller than the real one. ${ }^{[15,28,29]}$ For disperse DNA molecules, forces within the entropic regime are sufficient to almost fully stretch the complexes, and therefore the real contour length can be determined with high accuracy. To the best of our knowledge, this is the first work that

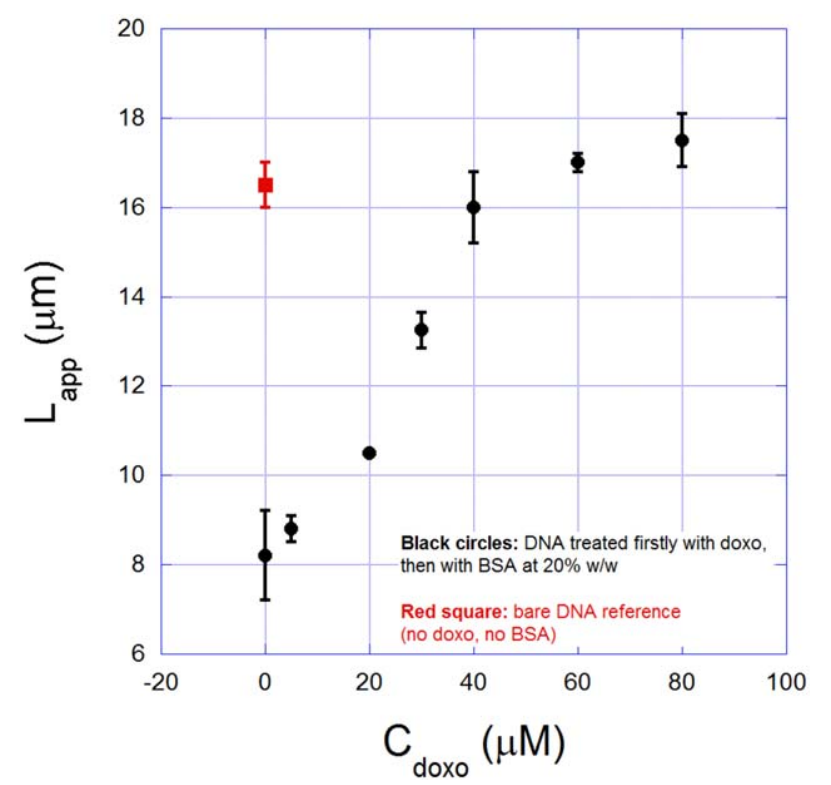

FIGURE 1 Black circles: apparent contour length $L_{\text {app }}$ of the complexes obtained firstly intercalating doxo in the disperse DNA molecules and then adding BSA. $L_{\text {app }}$ increases from $\sim 8.8 \mu \mathrm{m}$ (at $C_{\text {doxo }} \sim 5 \mu \mathrm{M}$ ) and reaches the saturation value $\sim 17.5 \mu \mathrm{m}$ (at $C_{\text {doxo }}$ $\sim 80 \mu \mathrm{M})$. The reference value obtained for the bare $\lambda$-DNA was also shown for comparison purposes (red square)

investigate the interaction of an intercalating drug with condensed DNA molecules at the single molecule level in the low-force entropic regime. Previously, this type of study was performed only in the highforce enthalpic regime. ${ }^{[14]}$

There are two types of experiments that can be performed to analyze the effects of BSA on the DNA-doxo interaction: (a) one can firstly intercalate doxo in disperse DNA molecules and then try to condense those DNA-doxo complexes adding BSA. (b) Alternatively, one can firstly use BSA to condense bare DNA and then use doxo in order to evaluate the effects of the drug on the condensates previously formed. These two possibilities can give very different results depending on the condensing agent, as recently demonstrated by Rocha et al. by using the tetravalent amine spermine and the neutral polymer polyethylene glycol (PEG) as condensing agents and ethidium bromide as the intercalator. ${ }^{[14]}$ Thus, the two possibilities were both analyzed here for the system DNA-doxo-BSA. The first type of experiment gives insights on how the changes of the mechanical properties of the DNA molecule induced by intercalators affect the DNA condensation process. The second type of experiment, on the other hand, is closer to the in vivo situation, in which one usually have condensed DNA molecules that interact with drugs.

\section{RESULTS AND DISCUSSION}

In Figure 1 (black circles), we show the apparent contour length $L_{\text {app }}$ of the complexes obtained firstly using doxo and then BSA. Since various different doxo concentrations were tested, each point in Figure 1 was obtained using a different $\lambda$-DNA molecule in a different sample 


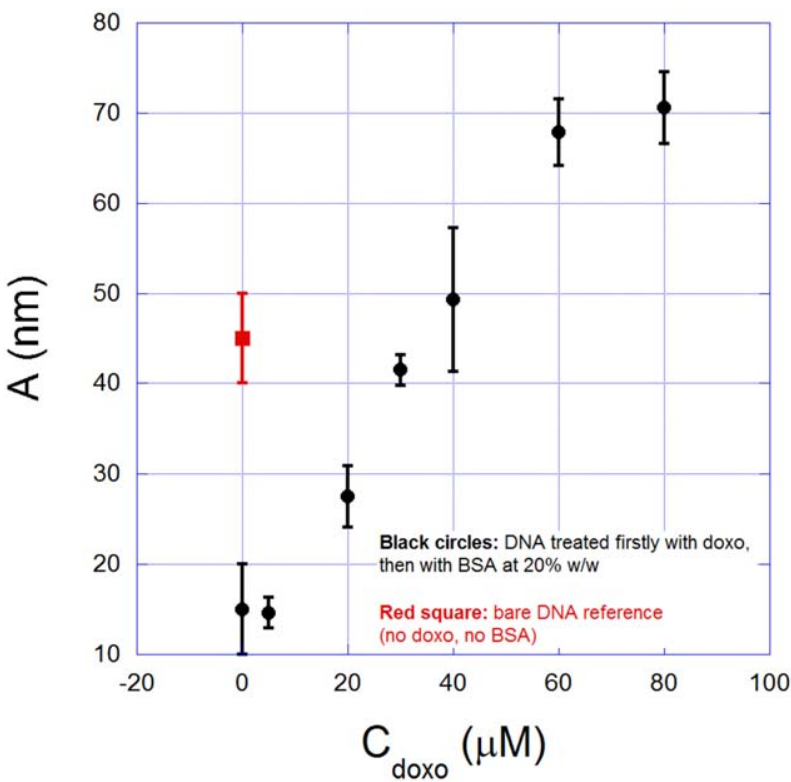

FIGURE 2 Black circles: persistence length A of the same complexes shown in Figure 1. This quantity decreases drastically from the bare DNA value (red square) when adding BSA in solution, and then increases monotonically with the doxo concentration (added before the BSA) until reaching the saturation value $\sim 70 \mathrm{~nm}$ at $C_{\text {doxo }} \sim 80 \mu \mathrm{M}$

chamber. Nevertheless, all bare DNA molecules were stretched many times before the addition of any compound in the sample, in order to guarantee that both contour and persistence lengths have the expected values (within the error bars, which are calculated here as the standard error of the mean for a series of seven different stretchings performed at each different concentration). In all measurements, the doxo concentration was varied, but the BSA concentration was always fixed at $20 \%$ $w t / w t$. The reference value obtained for the bare $\lambda$-DNA (without either doxo or BSA in the sample) was also shown for comparison purposes (red square). Observe that for $C_{\text {doxo }}=0$, the contour length decreases drastically from $\sim 16.5 \mu \mathrm{m}$ (the bare $\lambda$-DNA contour length) to the value $\sim 8.2 \mu \mathrm{m}$ when adding BSA in the buffer solution, due to the BSA-induced condensation of the DNA molecule. In other words, the contour length decreases by $\sim 50 \%$ when using only BSA (without doxo). This is the lowest value found for this parameter under our experimental conditions, as shown in Ref. ${ }^{[15]}$. Next, when we previously add doxo at a chosen concentration before adding BSA in the buffer, the DNA condensation process is still strong for low doxo concentrations, but is prevented for high doxo concentrations. Such conclusion can be achieved by noting that the apparent contour length increases from $\sim 8.8 \mu \mathrm{m}$ (at $C_{\text {doxo }} \sim 5 \mu \mathrm{M}$ ) and reaches the saturation value $\sim 17.5 \mu \mathrm{m}$ (at $C_{\text {doxo }} \sim 80 \mu \mathrm{M}$ ).

Figure 2 shows the results obtained for the persistence length $A$ of the same complexes. Observe that the qualitative behavior is the same one observed for the apparent contour length: the persistence decreases drastically for $C_{\text {doxo }}=0$ when adding BSA in solution, and then increases monotonically with the doxo concentration (added before the BSA) until reaching the saturation value $\sim 70 \mathrm{~nm}$ at $C_{\text {doxo }} \sim 80 \mu \mathrm{M}$.
The results of Figures 1 and 2 let us to conclude without doubt that doxo hinders the BSA-induced condensation process when previously intercalated in the DNA molecule. Such result agrees to those obtained for other types of DNA condensates and intercalators. In fact, Rocha et al. have recently shown that the intercalator ethidium bromide prevents DNA condensation induced both by the cationic ligand spermine and by the neutral polymer PEG when previously intercalated at high concentrations. ${ }^{[14]}$ Similar conclusions were also obtained by Widom and Baldwin ${ }^{[16]}$ and by the group of Yoshikawa ${ }^{[17,18]}$ by using various condensing agents and intercalators.

From the point of view of mechanical properties, the results discussed above can be understood remembering that intercalators in general increases both the DNA contour and persistence lengths, ${ }^{[30]}$ thus leaving the DNA longer and more rigid. Therefore, the energy necessary to fold and compact the resulting DNA-drug complex increases accordingly, which can hinder and even prevent the condensation process.

A relevant question that can be pointed here concerns the ability of doxo in unfolding DNA condensates previously formed by BSA. As mentioned before, we have also investigated this issue by inverting the order of application of the two compounds (doxo and BSA) in the sample preparation procedure. We show the results obtained in Figures 3 and 4. Observe that the qualitative behavior of both data is nearly similar to the one found when using doxo firstly. The major difference to the previous results (Figures 1 and 2 ) is the fact that the saturation appears to occur at higher concentrations when using BSA firstly $\left(C_{\text {doxo }}\right.$ $\sim 120 \mu$ M). Such result was expected because the intercalation of drug molecules in the double-helix in general occurs more efficiently in disperse DNA than in condensed DNA, since the binding sites are more

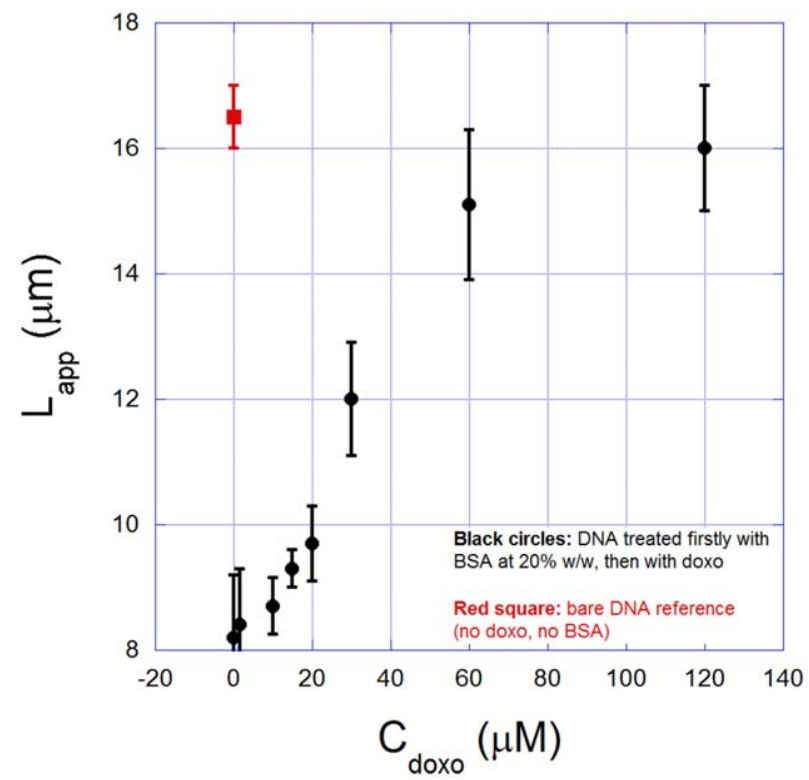

FIGURE 3 Black circles: apparent contour length $L_{\text {app }}$ of the complexes obtained firstly condensing the DNA molecule with BSA and then adding doxo. $L_{\text {app }}$ increases from $\sim 8.2 \mu \mathrm{m}$ at $C_{\text {doxo }} \sim 5$ $\mu \mathrm{M}$ and reaches the saturation value $\sim 16 \mu \mathrm{m}$ at $C_{\text {doxo }} \sim 120 \mu \mathrm{M}$. The reference value obtained for the bare $\lambda$-DNA was also shown for comparison purposes (red square) 
${ }^{4 \text { of } 7}$ W I LEY Biopolymers

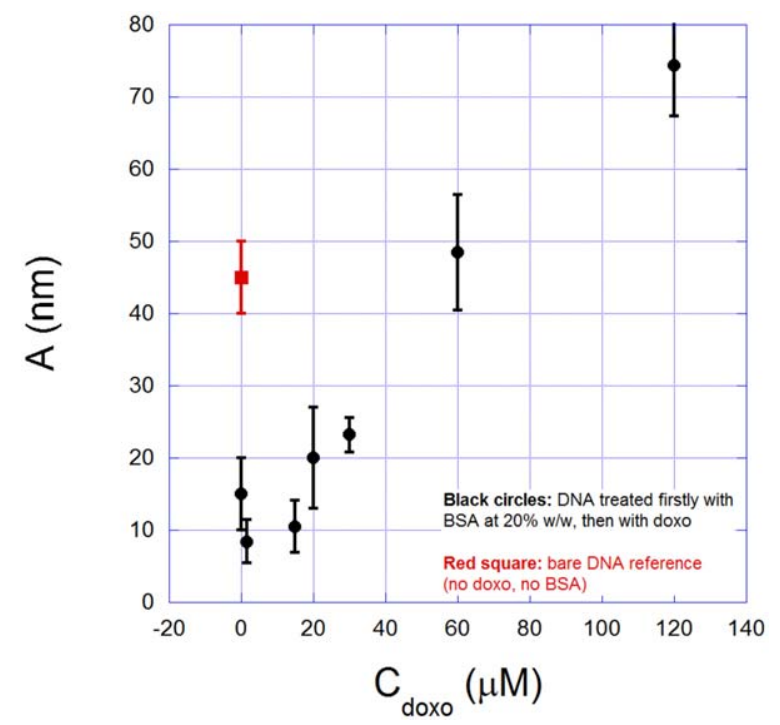

FIGURE 4 Black circles: persistence length $A$ of the same complexes shown in Figure 3 . This quantity decreases drastically for $C_{\text {doxo }}=0$ when adding BSA in solution and then increases monotonically with the doxo concentration (added after BSA) until reaching the saturation value $\sim 70 \mathrm{~nm}$ at $C_{\text {doxo }} \sim 120 \mu \mathrm{M}$

accessible in the former case. In fact, depending on the compaction degree imposed to the DNA molecule by a condensing agent, the interhelical spacing inside the condensates can be small enough to prevent the access of intercalator molecules. ${ }^{[14]}$ For the case of BSA under the experimental conditions of the present work, however, the results of Figures 3 and 4 suggest that the condensates can be well (but not completely) unfolded at $C_{\text {doxo }} \sim 120 \mu \mathrm{M}$, since the saturation value obtained for the contour length is $\sim 16 \mu \mathrm{m}$, therefore lower than the result obtained when using doxo firstly $(\sim 17.5 \mu \mathrm{m})$.

In summary, it was shown that the presence of previously intercalated doxo hinders the DNA condensation process induced by the

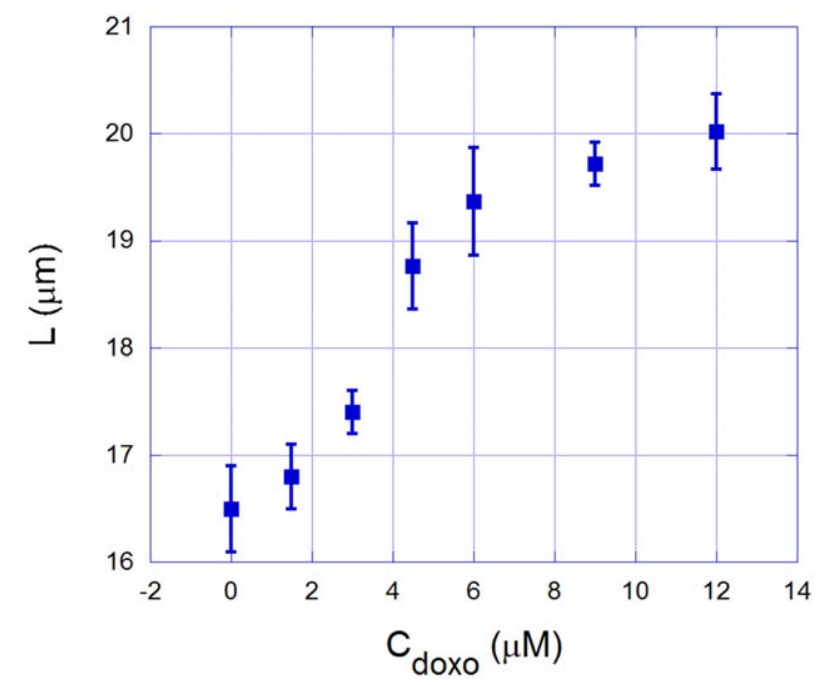

FIGURE 5 Contour length $L$ of the DNA-doxo complexes obtained in the PBS buffer without BSA. Observe that $L$ increases from the bare $\lambda$-DNA value $(\sim 16.5 \mu \mathrm{m})$ until the saturating value of $\sim 20 \mu \mathrm{m}$ obtained at $C_{\text {doxo }} \sim 12 \mu \mathrm{M}$

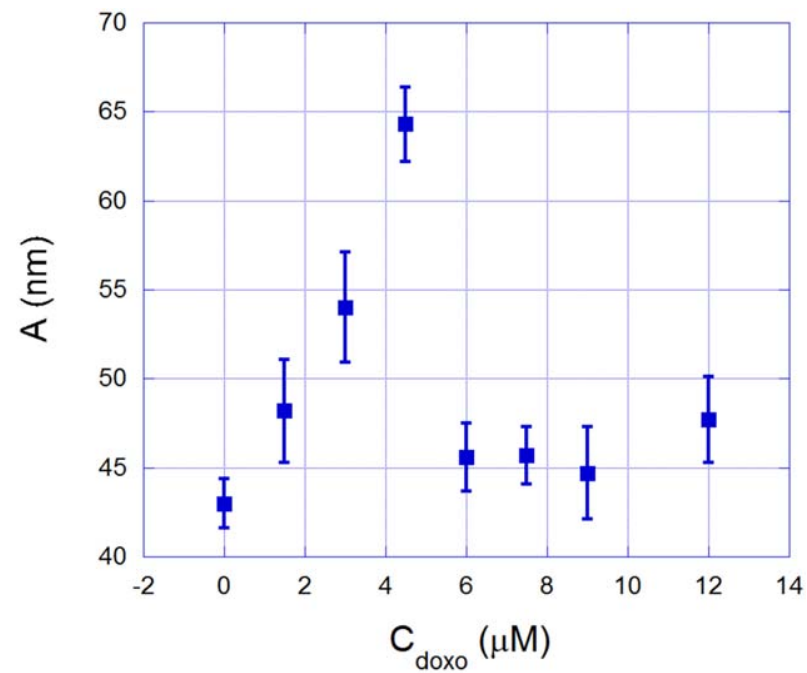

FIGURE 6 Persistence length $A$ of the same complexes shown in Figure 5. Observe that this quantity increases until $C_{\text {doxo }} \sim 4.5 \mu \mathrm{M}$ and then decreases abruptly for higher drug concentrations

protein BSA. On the other hand, when bare DNA is previously condensed by BSA, doxo is capable to considerably unfold these condensates at high drug concentrations. In any case, the interaction between doxo and the DNA molecule suffer changes when BSA is present in the buffer.

In order to advance on our analysis, one should compare the results presented above (Figures 1-4) to the equivalent results obtained for DNA-doxo complexes without BSA in the buffer solution, that is, when the DNA molecules are fully dispersed in solution. Recently, we have performed single molecule stretching on DNA-doxo complexes under approximately the same experimental conditions reported here (except by the presence of BSA), obtaining the results which are shown here in Figures 5 and 6. ${ }^{[21]}$

By comparing the results of Figures 1, 3, and 5, one should note that the qualitative behavior of the contour length as a function of doxo concentration is not affected by the presence of BSA, always increasing. Observe however that the saturation value of the contour length reached when BSA is present is considerably smaller than that found in the protein absence. In fact, when DNA is previously intercalated with doxo and then compacted with BSA (Figure 1) the saturated contour length found is $\sim 17.5 \mu \mathrm{m}$, while the value reported in Figure 5 for the DNA-doxo system without BSA is $\sim 20 \mu \mathrm{m}$. In order to investigate such difference, in the BSA experiments we have also measured the contour length value at the intermediate step, that is, after the addition of doxo and before adding BSA to the sample. For sufficiently high doxo concentrations ( $>12 \mu \mathrm{M})$, we found a contour length value at the intermediate step always close to $20 \mu \mathrm{m}$, in agreement with the data of Figure 5 . The measured decrease of the contour length obtained when adding BSA is due to the depletion forces induced by the protein on the DNA-doxo complexes and also due to a specific interaction between BSA and doxo, which changes the chemical equilibrium of the DNA-doxo interaction, as we discuss below.

Observe that the main difference between the results of Figures 1, 3 , and 5 is that, when BSA is present, one needs much higher doxo 
concentrations in order to achieve the contour length saturation. Such result implies that BSA, besides condensing the DNA molecules, also changes the chemical equilibrium between DNA and doxo, reducing the "effective affinity" between these two molecules, in the sense that one needs much more drug molecules in solution in order to saturate the available DNA binding sites. This reduction of the "effective affinity" was also confirmed by the persistence length data (see Figures 2, 4, and 6). In order to understand such effect, we have also investigated the existence of a direct interaction between doxo and BSA, which would compete with the DNA-doxo interaction studied in the optical tweezers experiments. Since a BSA-doxo interaction cannot be detected in such experiments, we have performed surface plasmon resonance (SPR) measurements with BSA and doxo in order to evaluate the interaction between the two compounds. Such experiments are described and discussed in detail in the Supporting Information. Briefly, we have found that there is a considerable interaction between BSA and doxo under our experimental conditions, obtaining the basic thermodynamic parameters of the interaction and the equilibrium association binding constant of the BSA-doxo interaction at ambient temperature $\left(25^{\circ} \mathrm{C}\right)$ : $K_{\mathrm{BSA}-\text { doxo }} \sim 4.8 \times 10^{3} \mathrm{M}^{-1}$. Such binding constant is about two orders of magnitude smaller than the constant obtained in Ref. 21 for the DNA-doxo interaction in the PBS buffer: $K_{\mathrm{DNA} \text {-doxo }}$ $\sim 2.7 \times 10^{5} \mathrm{M}^{-1}$.

Although the DNA-doxo binding affinity is much higher than that of the BSA-doxo binding, the concentration of BSA molecules in the sample chamber greatly exceeds the DNA concentration. In fact, in order to achieve DNA condensation, BSA was used at $20 \% \mathrm{wt} / \mathrm{wt}$, which corresponds to $\sim 3 \mathrm{mM}$. The DNA concentration used in the sample chamber in all experiments, on the other hand, was $\sim 4.8 \mu \mathrm{M}$, that is, approximately 1000 times smaller. Thus, when BSA is present in the buffer solution, we expect that the doxo concentration needed to saturate the DNA binding sites will considerably increase, since many doxo molecules will bind to BSA. As a first approximation, the doxo concentration needed to achieve the saturation binding in DNA should increase by $\sim C_{\mathrm{BSA}} K_{\mathrm{BSA}-\text { doxo }} / C_{\mathrm{DNA}} K_{\mathrm{DNA} \text {-doxo }} \sim 10$, that is, one needs to use $\sim 10 \times$ more doxo in solution when BSA is present at $20 \%$ $\mathrm{wt} / \mathrm{wt}$ in order to reach the saturation for the DNA-doxo interaction. Such estimation agrees well to the experimental results found for the mechanical parameters, since we have found that both persistence and contour lengths saturate at $\sim 12 \mu \mathrm{M}$ in the absence of BSA (see Figures 5 and 6) and at $\sim 100 \mu \mathrm{M}$ when BSA is present at $20 \%$ wt/wt (see Figures 1-4).

In addition, it is important to mention the possibility of existence of condensed and uncondensed regions on the DNA-doxo complexes, which may affect the local binding equilibria. Nevertheless, the DNA used in the experiments is considerably long $(48,502$ base pairs) such that what we measure is an average behavior of the drug along the double-helix. We were not able to discriminate different DNA regions using only force spectroscopy here: there is no evidence of different regions on the force-extension curves obtained. If the condensing agent used here was not a DNA depletant, AFM would be a possibility to investigate this issue in more detail. Nevertheless, the DNA condensation promoted by such type of condensing agent is strongly dependent on the concentrations of both the depletant and salt. In AFM, one needs to deposit the sample on a substrate and dry it in order to perform the imaging of the complexes. This drying procedure of the sample is somewhat problematic when using an agent that condenses the DNA via depletion interactions, since its concentration changes drastically when drying the sample, and the depletion interactions themselves only exist in solution.

Let us now compare the persistence length data obtained in the absence (Figure 6) and in the presence of BSA in solution (Figures 2 and 4). Observe that the behavior of this mechanical parameter is more complicated, being modified even qualitatively due to the presence of BSA. In fact, the presence of the protein in the buffer has prevented the abrupt decrease of this parameter shown in Figure 6. As discussed in Refs. 21,30, this abrupt decrease of the persistence length is probably due to the fact that the stretching force applied by the tweezers can induce a partial melting on the highly distorted double-helix structure of the DNA-doxo complexes, ${ }^{[31]}$ with the formation of denaturation bubbles that cause a decrease on the effective persistence length of the complexes. ${ }^{[32,33]}$ Therefore, when comparing the results of Figures 2, 4, and 6, one can conclude that the presence of BSA in the buffer stabilizes the double-helix structure of the DNA-doxo complexes, preventing partial denaturation at least when DNA is subjected to small forces $(F<3 \mathrm{pN})$. Such result has important biological implications. In fact, DNA molecules inside cells are typically subjected to very small forces, on the order of a few picoNewtons. ${ }^{[34]}$ Thus, the results obtained in the present work suggest that DNA molecules may be somewhat stabilized from denaturation in the crowded intracellular environment, which is important to maintain the genome integrity. As known, a considerable energetic cost (due to enzyme action) is needed in vivo to open and unzip the double-helix. In addition, it was previously demonstrated that the presence of BSA in the buffer suppresses DNA overstretching transition found at high forces $(\sim 65 \mathrm{pN}),{ }^{[15]}$ thus stabilizing the double-helix structure. Such result corroborates with the conclusion drawn above.

Finally, we now quantify the compaction degree of the DNA-doxo complexes for the different situations studied. One should note that both contour and persistence lengths influence the compaction degree of a polymer chain, since these parameters are related, respectively, to the linear size and bending rigidity of the polymer. Thus, the best parameter to describe the compaction degree of a polymer chain is the radius of gyration $R_{\mathrm{g}}$, which gives insights on the volume occupied by the macromolecule and depends on both its linear size and bending rigidity. For worm-like polymer chains, $R_{\mathrm{g}}$ can be written as a function of the contour $(L)$ and persistence $(A)$ lengths as

$$
R_{\mathrm{g}}=\sqrt{\frac{1}{3} A L\left(1-\frac{3 A}{L}+\ldots\right)} .
$$

Here, we calculate the compaction degree (also known as swelling degree) $\alpha$ as the relative change of the radius of gyration, that is,

$$
\alpha=\frac{R_{\mathrm{g}}-R_{\mathrm{g}}(0)}{R_{\mathrm{g}}(0)},
$$

where $R_{\mathrm{g}}(0)$ is the radius of gyration of the disperse bare DNA. 


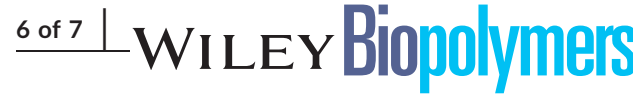

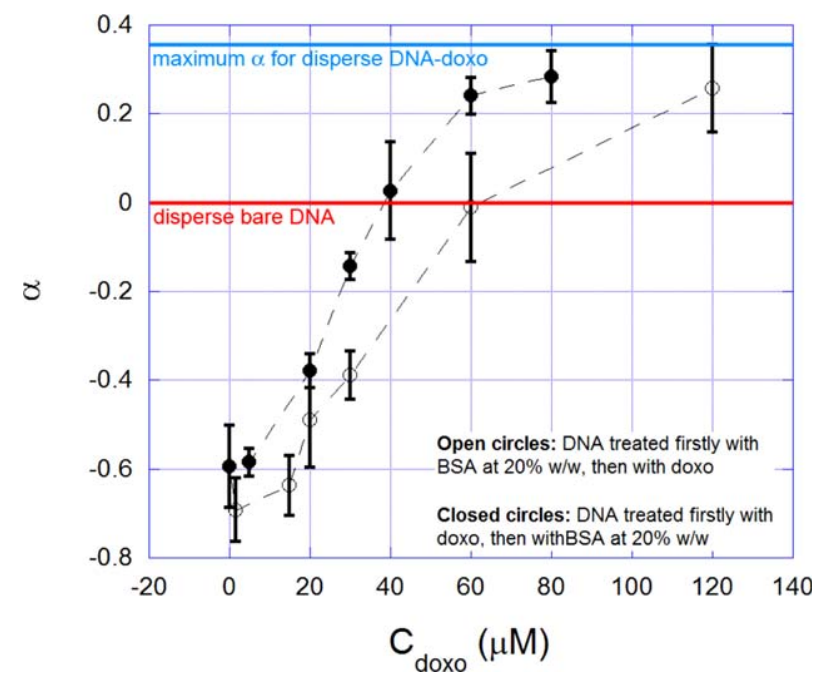

FIGURE 7 Compaction degree $\alpha=\left(R_{\mathrm{g}}-R_{\mathrm{g}}(0)\right) / R_{\mathrm{g}}(0)$ as a function of the doxo concentration in the sample. Open circles: DNA treated firstly with BSA at $20 \% \mathrm{wt} / \mathrm{wt}$, then with doxo. Closed circles: DNA treated firstly with doxo, then with BSA at $20 \% \mathrm{wt} / \mathrm{wt}$. Dashed lines are guides to the eyes. Horizontal red line highlights the value $\alpha=0$ corresponding to disperse bare DNA molecules. Horizontal blue line highlights the maximum value of $\alpha$ obtained for disperse DNA-doxo complexes ( 35\%)

For our experiments, $\alpha$ can be promptly calculated from the data of Figures 1 to 4 . In Figure 7, we show this parameter as a function of the doxo concentration in the sample, obtained for the two types of situations investigated. Two reference values of $\alpha$ are shown in the figure as horizontal lines. Horizontal red line: disperse bare DNA $(\alpha=0)$. Horizontal blue line: maximum value of $\alpha$ obtained for disperse DNAdoxo complexes ( 35\%). This reference value was calculated from the data of Figures 5 and 6.

Observe that for low doxo concentrations we have $\alpha<0$, indicating that the complex is compacted in relation to disperse bare DNA. Note also that the minimum compaction degree reached under our experimental conditions was $\sim-60 \%$. On the other hand, for sufficiently high doxo concentrations, we have $\alpha>0$, indicating that the complex has a radius of gyration higher than the disperse bare DNA. This is due to the high intercalated doxo concentration, which promotes an increase in both contour and persistence lengths of the DNA molecule, as discussed. Nevertheless, the complexes still have a radius of gyration smaller than the maximum value obtained for the disperse DNA-doxo complexes (horizontal blue line in Figure 7). Such result indicates that the presence of BSA in the buffer still promotes a certain degree of compaction for the DNA-doxo complexes, even for very high drug concentrations.

\section{4 | CONCLUSION}

In summary, the presence of the BSA protein in the buffer mimics two important effects present in the intracellular environment: DNA condensation and effective depletion interactions around the biopolymer due to the presence of various other macromolecules. In fact, such effects are not independent in the present case, since depletion interactions are behind the DNA compaction induced by the BSA protein. ${ }^{[20]}$ BSA is a charged globular protein which resembles many of the macromolecules present in the crowded intracellular environment. Thus, its use in buffer solutions instead of neutral linear polymers such as PEG in principle can give more precise insights on the mechanical behavior of the DNA molecule and on its interactions with chemotherapeutic drugs, simulating an environment closer to the in vivo one. Thus, we believe that the results of the present work advance in the understanding of the interactions between DNA and intercalators in vivo. In addition, our results evidence that caution is needed when adding proteins to the buffer used in experiments that intend to investigate DNA interactions with intercalators, since specific proteinintercalator binding can occur, changing the chemical equilibrium between the DNA molecule and the drug.

\section{ACKNOWLEDGMENTS}

This work was supported by the Brazilian agencies: Fundação de Amparo à Pesquisa do Estado de Minas Gerais (FAPEMIG), Conselho Nacional de Desenvolvimento Científico e Tecnológico (CNPq), and Coordenação de Aperfeiçoamento de Pessoal de Nível Superior (CAPES). The authors thank E. B. Ramos for helpful discussions and suggestions.

\section{AUTHOR CONTRIBUTIONS}

C. H. M. Lima performed the optical tweezers experiments. H. M. C. de Paula performed the SPR experiments. L. H. M. da Silva analyzed and interpreted the SPR experiments and wrote the Suppporting Information. M. S. Rocha designed the research, analyzed and interpreted the optical tweezers data and wrote the article.

\section{ORCID}

M. S. Rocha (iD http://orcid.org/0000-0003-0323-3718

\section{REFERENCES}

[1] K. R. Chaurasiya, T. Paramanathan, M. J. McCauley, M. C. Williams, Phys. Life Rev. 2010, 7, 299.

[2] M. S. Rocha, Integr. Biol. 2015, 7, 967.

[3] V. A. Parsegian, R. P. Rand, N. L. Fuller, D. C. Rau, Meth. Enzymol. 1986, 127, 400 .

[4] N. N. Degtyareva, B. D. Wallace, A. R. Bryant, K. M. Loo, J. T. Petty, Biophys. J. 2007, 92, 959.

[5] J. R. Neto, M. F. Colombo, Biopolymers 2000, 53, 46.

[6] A. L. Galo, J. R. Neto, D. P. Brognaro, R. C. Caetano, F. P. Souza, M. F. Colombo, J. Phys. Chem. B 2011, 115, 8883.

[7] J. E. Ladbury, Chem. Biol. 1996, 3, 973.

[8] X. Qu, J. B. Chaires, J. Am. Chem. Soc. 2001, 123, 1.

[9] F. A. P. Crisafuli, L. H. M. da Silva, G. M. D. Ferreira, E. B. Ramos, M. S. Rocha, Biopolymers 2016, 105, 227.

[10] Y. Murayama, Y. Sakamaki, M. Sano, Phys. Rev. Lett. 2003, 90 , 181021. 
[11] C. G. Baumann, V. A. Bloomfield, S. B. Smith, C. Bustamante, M. D. Wang, S. M. Block, Biophys. J. 2000, 78, 1965.

[12] J. Camunas-Soler, S. Frutos, C. V. Bizarro, S. de Lorenzo, M. E. Fuentes-Perez, R. Ramsch, S. Vilchez, C. Solans, F. Moreno-Herrero, F. Albericio, R. Eritja, E. Giralt, S. B. Dev, F. Ritort, ACS Nano 2013, 7, 5102.

[13] A. Lee, A. Karcz, R. Akman, T. Zheng, S. Kwon, S. T. Chou, S. Sucayan, L. J. Tricoli, J. M. Hustedt, Q. Leng, J. D. Kahn, A. J. J. J. S. Mixson, Angew. Chem. Int. Ed. 2014, 53, 10631.

[14] M. S. Rocha, A. G. Cavalcante, R. Silva, E. B. Ramos, J. Phys. Chem. B 2014, 118, 4832.

[15] C. H. M. Lima, M. S. Rocha, E. B. Ramos, J. Chem. Phys. 2017, 146, 054901.

[16] J. Widom, R. L. Baldwin, Biopolymers 1983, 22, 1621.

[17] Y. Yoshikawa, K. Yoshikawa, T. Kanbe, Biophys. Chem. 1996, 61, 93.

[18] N. Yoshinaga, T. Akitaya, K. Yoshikawa, Biochem. Biophys. Res. Commun. 2001, 286, 264.

[19] L. D. Murphy, S. B. Zimmerman, Biophys. Chem. 1995, 57, 71.

[20] K. Yoshikawa, S. Hirota, N. Makita, Y. Yoshikawa, Phys. Chem. Lett. 2010, 1, 1763

[21] E. F. Silva, R. F. Bazoni, E. B. Ramos, M. S. Rocha, Biopolymers 2017, 107, e22998.

[22] D. Salerno, D. Brogioli, V. Cassina, D. Turchi, G. L. Beretta, D. Seruggia, R. Ziano, F. Zunino, F. Mantegazza, Nuc. Acids Res. 2010, 38, 7089.

[23] C. Pérez-Arnaiz, N. Busto, J. M. Leal, B. García, J. Phys. Chem. B 2014, 118, 1288.

[24] L. B. Liao, H. Y. Zhoub, X. M. Xiao, J. Mol. Struct. 2005, 749, 108.

[25] D. Agudelo, P. Bourassa, G. Bérubé, H. Tajmir-Riahi, Int. J. Biol. Macromol. $2014,66,144$
[26] M. Airoldi, G. Barone, G. Gennaro, A. M. Giuliani, M. Giustini, Biochemistry 2014, 53, 2197.

[27] J. F. Marko, E. D. Siggia, Macromolecules 1995, 28, 8759.

[28] F. A. P. Crisafuli, E. C. Cesconetto, E. B. Ramos, M. S. Rocha, Integr. Biol. 2012, 2012, 568

[29] E. F. Silva, E. B. Ramos, M. S. Rocha, J. Phys. Chem. B 2013, 117, 7292.

[30] R. F. Bazoni, C. H. M. Lima, E. B. Ramos, M. S. Rocha, Soft Matter 2015, DOI: 10.1039/C5SM00706B.

[31] T. Hwa, E. Marinari, K. Sneppen, L. Tang, Proc. Natl. Acad. Sci. USA 2003, 100, 4411

[32] A. K. Dasanna, N. Destainville, J. Palmeri, M. Manghi, Phys. Rev. E 2013, 87, 052703

[33] F. Sicard, N. Destainville, M. Manghi, J. Chem. Phys. 2015, 142, 034903.

[34] R. Blossey, H. Schiessel, FEBS J. 2011, 278, 3619.

\section{SUPPORTING INFORMATION}

Additional Supporting Information may be found online in the supporting information tab for this article.

How to cite this article: Lima CHM, de Paula HMC, da Silva LHM, Rocha MS. Doxorubicin hinders DNA condensation promoted by the protein bovine serum albumin (BSA). Biopolymers. 2017;107:e23071. https://doi.org/10.1002/bip.23071 